(2) Open Access Full Text Article

REVIEW

\title{
New technology update: femtosecond laser in cataract surgery
}

\author{
This article was published in the following Dove Press journal: \\ Clinical Ophthalmology \\ 18 June 2014 \\ Number of times this article has been viewed
}

\section{Zoltan Z Nagy}

Department of Ophthalmology, Semmelweis University, Budapest. Hungary
Correspondence: Zoltan Z Nagy Department of Ophthalmology, Semmelweis University, Maria u 39, Budapest, Hungary

Email zoltan.nagy100@gmail.com
Abstract: Femtosecond lasers represent a new frontier in cataract surgery. Since their introduction and first human treatment in 2008, a lot of new developments have been achieved. In this review article, the physical principle of femtolasers is discussed, together with the indications and side effects of the method in cataract surgery. The most important clinical results are also presented regarding capsulotomy, fragmentation of the crystalline lens, corneal wound creation, and refractive results. Safety issues such as endothelial and macular changes are also discussed. The most important advantage of femtolaser cataract technology at present is that all the important surgical steps of cataract surgery can be planned and customized, delivering unparalleled accuracy, repeatability, and consistency in surgical results. The advantages of premium lenses can be maximally used in visual and presbyopia restoration as well. The advantages of premium lenses can be maximally used, not only in visual, but in presbyopia restoration as well. Quality of vision can be improved with less posterior chamber lens (PCL) tilt, more centralized position of the PCL, possibly less endothelial damage, less macular edema, and less posterior capsule opacification (PCO) formation. This technological achievement should be followed by other technical developments in the lens industry. Hopefully this review article will help us to understand the technology and the results to demonstrate the differences between the use of femtolasers and phacoemulsification-based cataract surgery. The most important data of the literature are summarized to show ophthalmologists the benefits of the technology in order to provide the best refractive results to the patient.

Keywords: femtosecond laser-assisted cataract surgery, capsulotomy, lens fragmentation, corneal wound, arcuate keratotomy, safety, consistency of results

\section{Introduction}

Nowadays, cataract surgery is the most commonly performed ophthalmic procedure. It is estimated by the World Health Organization (WHO) that approximately 18 million cataract procedures are performed globally every year, which will increase to 24 million soon due to demographic changes, the aging population, and changes in indication for crystalline lens surgery. ${ }^{1}$ These days, cataract surgery is not only a vision restoration procedure, but also a refractive type of operation. Ophthalmic surgeons restore the clarity of the optical media and change the refraction of the patients as well. Moreover, presbyopia treatment is also an option for patients. Usually, patients have better vision quality after the lens procedure than before developing the cataract. As phacoemulsification became a safer and more established procedure, ophthalmologists have operated on younger and younger patients. With younger age, the expectation of patients increases. Therefore, new technologies and surgical techniques are also needed - one of these is the development of laser technology. Ophthalmologists 
use different laser equipment during their everyday practice. The latest development is the introduction of femtolaser technology in cataract surgery.

Different lasers have been used in ophthalmology for more than 50 years. Ophthalmology has always had a pioneering role in laser use. Different lasers operate with specific wavelength, pulse pattern, pulse energy, pulse duration, repetition rate, and spot size. According to these parameters, they are absorbed in different tissues at different depths and the biological effect also varies. Today, there is no tissue within the eye that cannot be treated with some type of laser. The laser-tissue interaction can be photothermal or photochemical. The other distinctions are thermal effects, such as photocoagulation (eg, argon laser), photoablative effect (eg, excimer lasers), or photodisruption (eg, yttrium aluminium garnet [YAG] laser).

The Nd:YAG (neodymium-doped YAG) lasers and femtosecond lasers operate at similar wavelengths in the infrared range of the electromagnetic spectrum. On the other hand, the tissue effect is different, because femtosecond lasers operate with extremely short pulse durations without thermal effects. A femtosecond is one-quadrillionth, or in other words $10^{-15}$ of a second, while the Nd:YAG laser pulse duration is in the nanosecond range, ie, $10^{-9}$.

\section{Femtosecond lasers in ophthalmology}

Femtosecond lasers appeared first in corneal surgery to create the corneal flaps for refractive surgeons. Femtosecond lasers have been introduced first in corneal surgery, to create the corneal flaps. The first corneal femtolasers operated at $30 \mathrm{kHz}$, then the repetition rate was doubled; the latest $150 \mathrm{kHz}$ femtolasers are able to create a corneal flap within 10 seconds. The higher the repetition rate, the lower the energy required to obtain the same tissue effect.

Femtosecond lasers in cataract surgery use a pulse duration of 400-800 fs; the energy range is in $\mu \mathrm{J}$, which is usually less than in YAG capsulotomy, in which the surgeon uses $1-3 \mu \mathrm{J}$. During surgery of the crystalline lens, femtosecond laser energy can be increased maximally to $10-15 \mu \mathrm{J}$.

The photodisruption effect is achieved when the sharply focused beam of the femtosecond laser hits the tissue with ultrashort duration and generates plasma within the affected tissue. The plasma expands at high speed in a shock wave form and displaces the surrounding tissues. With time, the plasma cools down and so-called cavitation bubbles are formed. At the tissue level, photodisruption occurs within the laser's focal point without any heat development or damage to the collateral tissues. Based on the photodisruption principle, femtolasers for cataract surgery can create tissue separation and very precise cuts within the cornea, the lens capsule, and within the crystalline lens.

The numerical apertures of different femtolasers are very important characteristics during corneal and lens use. The numerical aperture affects the spot size and volume significantly. The higher the numerical aperture, the less dispersion in the laser beam (better focused laser beam) and the lower the energy needed to provide the same effect. The precision of the depth of the cut is also better. Therefore, corneal treatments need a higher numerical aperture and lower energy, while the crystalline lens needs a low numerical aperture with higher energy level. Femtosecond lasers can have a repetition rate up to $160 \mathrm{kHz}$. It is very important that a femtosecond laser treating the cornea and the crystalline lens simultaneously should have great flexibility in pulse energy, pattern, duration, and repetition rate.

Based on the initial results and experiences, the US Food and Drug Administration (FDA) cleared the four main steps of femtosecond laser-assisted cataract surgery (FLACS) in 2009 for the LenSx ${ }^{\circledR}$ (Alcon Laboratories, Inc., Fort Worth, TX, USA) femtolaser-like capsulorhexis, lens fragmentation (liquefaction), corneal incisions, and arcuate incisions. Other companies have developed different femtolasers for cataract surgery, including Abbott Medical Optics Inc., (CATALYS ${ }^{\circledR}$, Abbott Medical Optics Inc., Santa Ana, CA, USA), the LensAR (LENSAR Inc., Orlando, FL, USA), and the Victus ${ }^{\circledR}$ (TECHNOLAS Perfect Vision GmbH, Munich, Germany; Bausch \& Lomb Incorporated, Rochester, NY, USA), which appeared on the market and the companies received FDA clearance for their femtolasers following LenSx. LenSx was acquired by Alcon Laboratories Inc. in 2011 and became Alcon-LenSx. With the different companies, the physical principle of femtosecond lasers is very similar. The main difference is the type of patient interface: curved interface with a soft contact lens (Alcon-LenSx) or fluid interface (Victus, Abbott Medical Optics Inc., LensAR). The other difference is in the imaging systems, the LenSx machine uses optical coherence tomography (OCT) imaging (Figures 1 and 2), while the others use Scheimpflug imaging.

Nowadays, patients expect perfect postoperative visual acuity following cataract surgery with excellent visual quality and spectacle independency regarding far and near vision. Femtolaser technology offers automated steps during the critical phase of cataract surgery with consistent results, which increase predictability. 


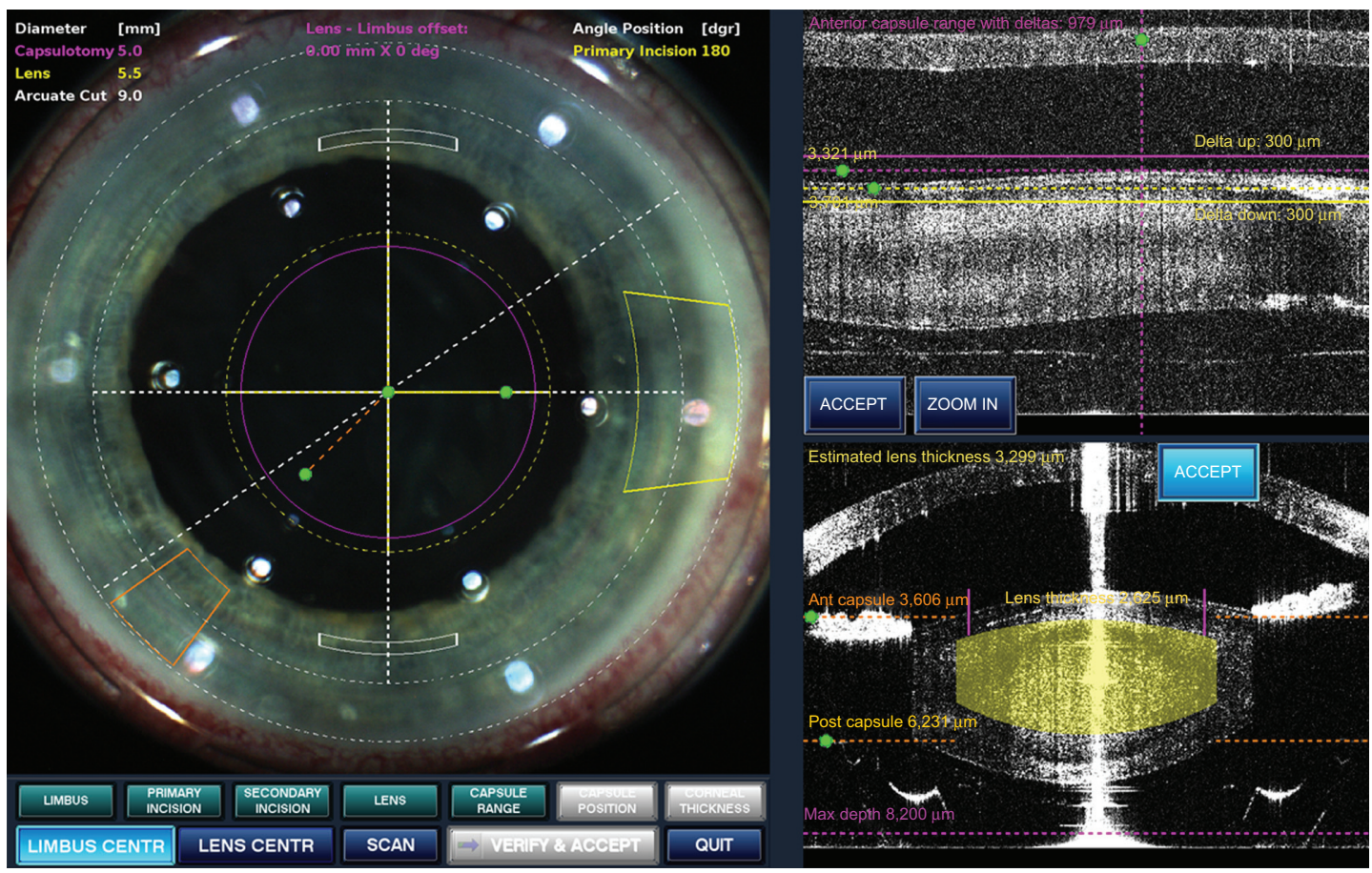

Figure I The screen for the surgeon. Note the corneal wounds and the astigmatic incisions. On the upper right, the OCT identifies the endothelial layer, the anterior capsule (highest and lowest point); on the lower part of the image, OCT identifies the cut within the crystalline lens (yellow area).

Abbreviation: OCT, optical coherence tomography.

Since the first $510 \mathrm{kHz}$ femtosecond laser (Alcon-LenSx) received FDA clearance in 2009, femtosecond lasers have made their grand entrance into the field of cataract surgery. Ophthalmologists and ophthalmic practices equipped with this new technology are able to improve their surgical results and the steps of cataract surgery by perfecting some of the most difficult parts of the procedure, including capsulorhexis, nuclear fragmentation, and corneal incisions. The automation which is based on the femtolaser technology can provide consistent and customizable results with micrometer precision in creating the capsulorhexis and in pre-cutting the nucleus, reducing the overall energy needed to remove the cataract. The femtosecond lasers provide more accurate and reproducible results compared to manual techniques. The main aim is not for ophthalmic technicians or optometrists to take over the surgery from ophthalmologists but to help ophthalmologists during the crucial steps of cataract surgery.

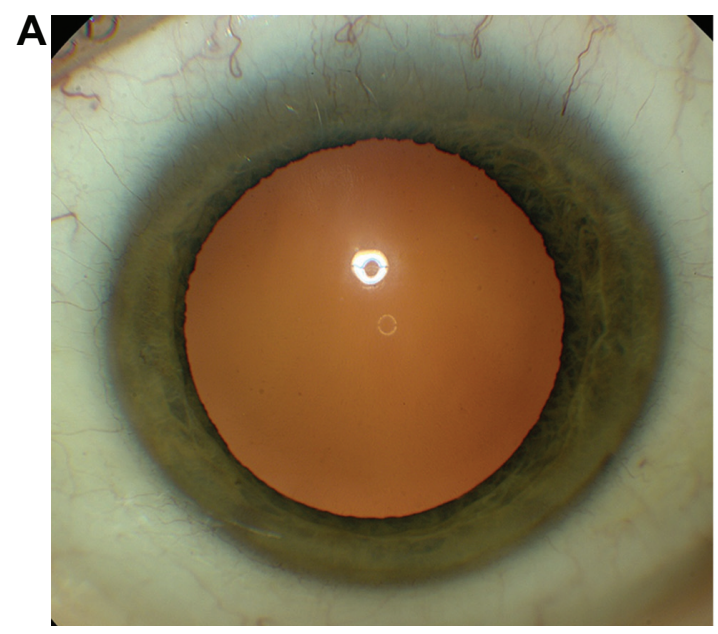

B

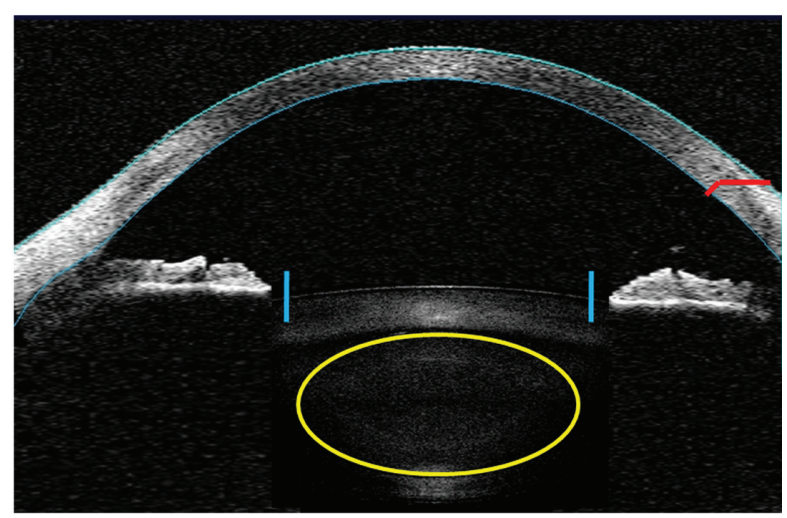

Figure 2 The proprietary image-guided system (A) allows the surgeon to take a preoperative OCT image (B) and position the planned incisions and photolysis patterns on the patient's eye. The blue and yellow overlays represent the lens photolysis and capsulotomy patterns (B). The red represents the corneal incisions (B). The size and position of all patterns can be preprogrammed and adjusted for ultimate surgeon control.

Abbreviation: OCT, optical coherence tomography. 
The first ever human femtolaser-assisted cataract operation was performed in 2008 in the Department of Ophthalmology, Semmelweis University, Budapest, Hungary. The first experiences were reported in peer-reviewed ophthalmic journals. ${ }^{2-18}$ In this review article, the results of the first team and others will be presented.

\section{Most important indications of femtolaser cataract surgery}

- Anterior capsulotomy

- Laser fragmentation of the crystalline lens (harder lenses)

- Laser liquefaction of the crystalline lens (soft lenses)

- Single plane or multiplane (uniplanar, biplanar, triplanar, etc) corneal cuts with 2-3 incisions

- Arcuate corneal cuts to control preoperative corneal astigmatism

- Clinical indications:

O Cataract

O Refractive lens exchange (RLE)

- Traumatic cataract (rupture of the anterior capsule)

○ Zonular dehiscence (trauma, Marfan syndrome)

○ Angle-closure glaucoma (narrow anterior chamber)

○ Pseudoexfoliation syndrome

O Pediatric cataract (for anterior and posterior capsulotomy)

\section{Contraindications (relative)}

- Small, non-dilating pupil

The only relative contraindication is a non-dilating pupil that is less than $5.0 \mathrm{~mm}$ in diameter (optimally it should be larger than $6.0 \mathrm{~mm}$ ). Capsulotomy is possible in the case of a $5.0 \mathrm{~mm}$ diameter central pupillary area, but because the edge of the iris is within $1.0 \mathrm{~mm}$, the chance of hitting the pupillary edge is high; the pupil may be narrower at commencing phacoemulsification than expected. Smaller capsulotomy can also be performed, but the chance of capsular phimosis is increased if capsulotomy diameter is less than $4.0 \mathrm{~mm}$. For non-dilating pupils, Malyugin rings offer a good solution. ${ }^{17}$ The ring should be placed into the anterior chamber, the viscoelastics removed, and the wound temporarily sutured with a 10/0 nylon. After performing capsulorhexis and fragmentation, surgery is similar to normal dilated cases. ${ }^{17}$

\section{Sequence of the femtolaser procedure}

- Patient selection

- Consenting
- Patient head positioning

- Drop anesthesia

- Capsulorhexis

- Lens liquefaction or fragmentation

- Astigmatism correction (arcuate incisions at 80\% depth)

- Corneal wound

\section{Treatment parameters}

During femtolaser cataract surgery, the surgeon is able to modify all treatment parameters, ie, can change the diameter of capsulotomy between 4.5 and $6.0 \mathrm{~mm}$ (accommodating lenses usually need larger diameter of capsulotomy compared to nonaccommodating monofocal or multifocal lenses). For lens fragmentation today, the so-called hybrid pattern is recommended: the central $3.0 \mathrm{~mm}$ core is liquefied and the peripheral parts fragmented into $4-8$ cuts (cross pattern and cake or pizza pattern). This pattern allows the surgeon to remove the central lens part easily and gives access to the peripheral parts, reducing the ultrasonic phaco energy and time. This technique allows better visual acuity on the next day postoperatively, reduced corneal edema, and reduced cystoid macular edema (CME). Among our patients, reduced retinal thickness compared to manual phacoemulsification cases was noted, with presumably less phaco energy due to femtolaser pretreatment. An additional benefit may be less manipulation within the eye thanks to a pre-fragmented nucleus. So far, lens fragmentation has been performed in all kinds of cataracts, from soft to very hard lenses. Presently, it is recommended for cataracts up to +4.0 grade. In brunescent and white tumescent cataracts, the laser absorption is not perfect. In the latter cases, capsulotomy and corneal incisions provide the most important benefits of this technology. Further developments are needed to make lens fragmentation more effective in such cases.

\section{Ergonomics}

For the femtolaser equipment, a $11 \times 14$ feet $(3.3 \times 4.2 \mathrm{~m})$ room dimension is recommended for ergonomic use. In case of a larger room, the femtolaser can be placed into the same room as the phacoemulsification device.

\section{Planned series of procedures}

Firstly, the capsulotomy should be performed, followed by fragmentation/liquefaction, and lastly corneal cuts. The reasoning behind this is that during lens fragmentation, gas bubbles may appear within the crystalline lens, which could elevate the anterior capsule. If this happens, another OCT measurement is required, in which case the treatment parameters will need to be redesigned; therefore, 
capsulotomy should be the first to be carried out. Small gas bubbles freely move up to the endothelial layer, but this does not prevent effective lens fragmentation. Corneal cuts are performed lastly because they are performed from the inside to the outside. Previous gas bubbles do not alter the anatomical parameters of the cornea.

In most cases, surgeons can manually achieve a perfect rhexis, but the patients may inadvertently move, rendering the rhexis imperfect. Younger patients are more anxious about premium lens implantation and are more likely to move during surgery. This can lead to an imperfect capsulorhexis, causing lens tilt, which inevitably leads to an increase in higher order aberrations, leaving the patients with a postoperative visual acuity that is not as good as it could be. Therefore, even high-volume cataract surgeons need a reliable technology achieving attainable, consistent results, especially when advanced technology intraocular lenses (IOLs; multifocal, accommodative, aspheric, toric, toric-multifocal, etc) are used. The new femtosecond laser technology may offer these advantages.

\section{Possible post-femtolaser surgical findings and complications Subconjunctival redness or hemorrhage}

Usually, mild-to-moderate redness and hemorrhages can be noted, especially in patients on anticoagulant therapy who are prone to developing conjunctival redness. Patients readily accept this phenomenon if given proper preoperative information. Redness usually resolves quickly. Conjunctival redness can also be decreased by lowering the suction force exerted by the patient interface..$^{14,19,20}$

\section{Pupillary constriction}

Pupillary constriction was a frequent problem during the early years of femtosecond laser cataract surgery. This is caused by the suction force and bubbles may appear in the anterior chamber, producing small amounts of free radicals. Preoperatively, the pupil should be at least $6.0 \mathrm{~mm}$ in diameter. Shock waves from laser pulses can be close to the iris, especially in cases where the iris is not well dilated, which can cause inadvertent miosis. Preoperatively, more dilating agents are advised, as well as nonsteroidal anti-inflammatory drugs (NSAIDs; eg diclofenac drops). During laser programming, the capsulotomy diameter should be at least $1.0 \mathrm{~mm}$ smaller than the pupillary diameter. ${ }^{14}$ Surgeons should wait as short a time as possible between femtolaser pretreatment and cataract surgery (5-10 minutes is recommended). In normal, well-dilated cases, pretreatment in 2-3 patients is achievable, but if possible, one femtolaser followed by one cataract surgery is the preferred pattern. In non-dilating cases, a Malyugin ring is a good solution for performing femtolaser pretreatment. ${ }^{17}$

\section{Capsular blockage syndrome}

Intraoperative capsular blockage syndrome was first reported in 2011 during hydrodissection. ${ }^{21}$ Large diameter hydrodissection cannula with high-speed fluid egress may impede gas bubbles from leaving the nucleus. The consequent increase in pressure within the lens can cause rupture of the posterior capsule, with consequent sinking of the nucleus into the vitreous cavity. The so-called "rock-and-roll" technique according to Nagy helps to avoid this possible complication, ie, after meticulous, slow, and gentle hydrodissection, the surgeon should press down (to "rock") the nucleus a bit and move it (to "roll"). ${ }^{14}$ This suggested modification of the surgical technique (titrated injection of hydrodissection fluid and meticulously splitting the nucleus) helps to release intralenticular gas bubbles and to avoid this threatening complication. With respect to the differences in femtolaser cataract surgery, by admitting the importance of the learning curve and keeping the above mentioned principles, the feared capsular blockage syndrome can be avoided. After the first report, surgeons became more cautious and are now able to prevent blockage syndrome.

\section{Corneal incision sizing and position}

Femtolaser-created incision widths may be tighter than expected; therefore, it is recommended to stretch the edges of the incision with fine hand movements using the opening spatula. ${ }^{9,12,18}$ At programming, surgeons should note the proper centration of the patient interface. If the patient interface is not centered perfectly, the actual corneal wounds are more central than intended, which might cause surgically induced astigmatism (SIA). Perfect docking is mandatory to avoid lens tilt and centrally shifted corneal incisions. In the latter case, lens capsulotomy and fragmentation may also be asymmetrical leading to partial capsulotomy and fragmentation. ${ }^{14}$

\section{Clinical results of femtolaser cataract surgery} Anterior capsulotomy

In the literature, the importance of lens displacement regarding postoperative refraction has been extensively studied. It has been shown that $1 \mathrm{~mm}$ anterior displacement of the posterior chamber lens (PCL) causes a 1.25 D myopic shift. In the case of a posterior displacement, hyperopia occurs with the same diopter magnitude. If the capsulotomy 
is too small, anterior capsular fibrosis (capsular phimosis) may occur, implanting a single-piece PCL. If the capsulorhexis is too large, not overlapping the PCL will cause tilt, decentration, an increase of higher order aberrations, optical aberrations, and posterior capsule opacification ( $\mathrm{PCO}){ }^{22-27}$

In spite of the most sophisticated lens calculation formula, effective lens position (ELPo) within the capsular bag is mostly dependent on capsular size, shape, and centration of the capsulotomy. Therefore, inaccuracy of ELPo is the major cause in IOL power calculation errors. ${ }^{28,29}$

Our team and others have established that femtosecond laser capsulotomies are more precise, consistent, and better centered compared with manual capsulorhexis., ${ }^{2,290}$ Better overlapping with a $0.25-0.5 \mathrm{~mm}$ anterior capsule over the PCL optics results in less tilt and decentration compared to manual continuous curvilinear capsulorhexis $(\mathrm{CCCs}) .^{2-4,11}$

Anterior capsulotomy was initially evaluated in ex vivo porcine eyes, in the first clinical series involving patients' eyes. The authors found that an intended $5.00 \mathrm{~mm}$ capsulorhexis in porcine eyes became $5.88 \pm 0.73 \mathrm{~mm}$ using the standard manual technique, and $5.02 \pm 0.04 \mathrm{~mm}$ using the femtolaser technology for capsulotomy. In human eyes, femtolaser technology achieved all capsulotomies within $\pm 0.25 \mathrm{~mm}$ exactness; on the other hand, with the manual technique, this was achieved in only $10 \%$ of the eyes. Using manual standard CCC technique, the diameter of the achieved capsulorhexis was on average within $\pm 1.00 \mathrm{~mm}$ of the intended diameter. $^{2}$

Capsulorhexis is very important in terms of the final refractive results of cataract surgery. Until now, the manual technique was the only available method. Therefore, not much was written about the significance of perfectly centered and guaranteed diameters of capsulorhexis. If the rhexis is larger than the intended diameter it could cause anterior or posterior shift or tilt of the implanted PCL. The ELPo is a very important issue in modern cataract surgery, especially with the advanced technology IOLs (AT-IOLs). ELPo is derived from the anatomical characteristics of the eye, such as the anterior chamber depth (ACD), the diameter of the capsulorhexis, and different IOL formulas. The key element is the size of the capsulorhexis. ${ }^{3}$ According to studies, a $1.0 \mathrm{~mm}$ difference in IOL position may result in an approximately $1.25 \mathrm{D}$ change in refractive error. A reproducible, well-centered, and properly positioned circular capsulorhexis that overlaps the optics of the IOL at $360^{\circ}$ is a prerequisite for good postoperative refraction, or in other words, predicting the refractive difference between the intended and achieved refraction. ${ }^{4}$ As mentioned in an earlier study, authors achieved the desired rhexis diameter in $100 \%$ of treated eyes using the femtolaser capsulotomy technique. ${ }^{2}$ Friedmann et al described similar accuracy in rhexis diameter and circularity performed by the femtosecond laser. ${ }^{30}$

In another prospective, nonrandomized clinical study in Hungary, 20 eyes were included that underwent $4.5 \mathrm{~mm}$ capsulotomies performed by the femtolaser (Alcon-LenSx); another 20 eyes received a $4.5 \mathrm{~mm}$ manual capsulorhexis. $\mathrm{ACD}$ and $\mathrm{AL}$ (axial length) were determined using the LenStar Optical Biometry (Haag-Streit AG, Koeniz, Switzerland) at 1 week, 1 month, and 1 year postoperatively. A significant difference in variability was found in the ACD:AL ratio and a significant difference was found between the two groups with reduced variability in the ELPo in femtolaser-treated eyes compared to the manual group during the whole follow-up period. ${ }^{3,4}$

A similar study was performed using different types of IOLs in a prospective single-surgeon study. The variability and predictability in ELPo and refractive outcomes were compared between femtolaser-treated and manually performed capsulorhexis eyes. During the study, monofocal (hydrophobic, acrylic one-piece) and multifocal (hydrophobic, acrylic one-piece) PCLs were implanted. Results showed better predictability and variability of ELPo for all types of IOLs if capsulotomy was performed by the femtolaser. In summary, it can be concluded that femtolaser capsulotomy has positively influenced the predictability of ELPo. ${ }^{11}$

\section{Anterior capsulotomy circularity and PCL centration}

Two studies were carried out in order to determine the exactness of circularity (how round is the capsulotomy?) and its effect on PCL centration postoperatively. The first studies showed that anterior capsulotomies performed using a femtosecond laser were more regularly shaped and showed better centration and a better capsule/IOL overlap compared to manual capsulorhexis..$^{2-4,11}$ The vertical diameter and horizontal IOL decentration of manual rhexes were statistically significantly higher. There were also significantly higher values of capsule overlap and better circularity values in the femtosecond laser-performed capsulotomies. Univariate analysis showed that the type of capsulorhexis (femtosecond or manual) $(P<0.01)$ and capsule overlap $(P=0.002)$ were significant predictors of horizontal decentration of IOLs. ${ }^{4}$

In another study, anterior capsulotomy was performed with the Alcon-LenSx femtosecond laser in 54 eyes and a manual CCC was performed in 57 eyes. The circularity and the exact area of the capsulotomy and IOL decentration were determined using Photoshop CS4 Extended 
(Adobe Photoshop Systems Inc., San Jose, CA, USA) 1 week and 1 month postoperatively. Circularity was statistically significantly better in the femtosecond laser group $(P=0.032)$ and there was significantly less incomplete overlap of capsulotomies in the manual rhexis group compared to the femtosecond-treated eyes $(28 \%$ of eyes versus $11 \%$; $P=0.033) .{ }^{11}$

\section{Type of fragmentation within the crystalline lens}

Femtosecond lasers offer different types of lens fragmentation. In soft lenses grading $<2.0$ nuclear cataracts according to the Lens Opacities Classification System (LOCS), a $5.0 \mathrm{~mm}$ central liquefaction is recommended, creating concentric rings (cylindrical pattern) within the nucleus of the crystalline lens. This possibility is especially important in RLE in high myopic and high hyperopic eyes and if the patient is over 45 years of age.

In nuclei grading $>2.0$, a fragmentation of the lens nucleus is recommended. This can be a "cross pattern" (two perpendicular incisions within the lens), or can be customized with an increased number of cuts. They are called "cake" or "pizza pattern" fragmentations (6-8 cuts). More cuts are not recommended, because during incisions, more fragmentation lines cannot be used effectively. At present, a hybrid pattern is preferred, using a central $3.0 \mathrm{~mm}$ diameter liquefaction and peripheral fragmentation lines. With this method, the surgeon is able to spare even more phaco energy and phaco time, thus increasing the safety of the method. Femtolaser fragmentation can be achieved effectively at present in +4.0 grade nuclear cataracts.

The "cubicle" pattern can also be used in order to minimize phacoemulsification energy. ${ }^{31}$

The liquefaction and fragmentation diameter area should not be more than $1.0 \mathrm{~mm}$ larger than the capsulorhexis diameter, because the back of the lens surface has a concave shape. In case of longer fragmentation lines, the possibility of harming the posterior capsule also increases. The inbuilt OCT provides perfect safety to control the distance from the posterior capsule. Presently, a 500-700 $\mu \mathrm{m}$ safety distance is recommended. The distance is usually set automatically by the femtolaser (LenSx femtolaser procedure). In some places, the fragmentation line can be closer than expected to the posterior capsule and so the surgeon should check it meticulously in case of crystalline lens tilt due to improper docking.

If the surgeon increases the length of the fragmentation line over the diameter of the capsulorhexis, due to the effective 500-700 $\mu \mathrm{m}$ safety distance from the peripheral posterior capsule, this distance could increase to $1.5 \mathrm{~mm}$ in the central part. If this is the case, effective incisions are difficult in the central part without using phacoemulsification and the advantage offered by the femtosecond laser could be lost. Therefore, the length of femtolaser fragmentation lines should not be much longer than the actual capsulotomy diameter.

In a prospective, non-randomized study, the authors evaluated the reduction of phacoemulsification time and power after femtosecond laser lens treatment and traditional phacoemulsification. Sixty eyes of 60 patients were included in each group. The mean cumulative dissipative energy (CDE) was significantly reduced in the femtosecond laser group and there was a $25 \%$ reduction in endothelial cell loss compared to the traditional manual phacoemulsification group. ${ }^{8}$

\section{Energy for phacoemulsification}

The comparison of femtosecond laser fragmentation with the cross pattern and the "quick chop" traditional phacoemulsification technique resulted in a $43 \%$ reduction in $\mathrm{CDE}$ and a $51 \%$ reduction in phacoemulsification time using the Infiniti (Alcon Laboratories Inc.) phacoemulsification machine. During the study, there were no complications either during the femtolaser pretreatment, nor during the phacoemulsification of the pre-fragmented nucleus. ${ }^{2} \mathrm{With}$ the newer fragmentation software, more CDE sparing is expected, increasing the safety of cataract removal regarding endothelial cells and CME.

Palanker reported a 39\% decrease in CDE using the Catalys system. Similar results have been reported by Conrad-Hengerer et al (29\% decrease in CDE). ${ }^{31,32}$

Depending on the cataract grade and fragmentation patterns, significant decreases in CDE and effective phaco time can be achieved by femtolaser technology, which in turn may increase the safety of the method regarding postoperative corneal swelling and loss of endothelial cells, etc. New randomized prospective studies are necessary to establish the real value of FLACS in fragmentation and long-term safety.

\section{Corneal wounds and arcuate incisions to control preoperative corneal astigmatism}

Corneal wounds with perfect structure and dimension are of utmost importance for controlling postoperative infection and to minimize SIA. Clear corneal non-sutured incisions have been reported to increase the rate of bacterial endophthalmitis. ${ }^{33}$ Square incisions were found to be more stable and caused less leakage. ${ }^{34}$ Wound characteristics also have an important role in IOL selection - especially for toric and multifocal PCLs. Manually blade-created wounds may have imprecise tunnel length and structure, and often require stromal 
hydration at the end of surgery. Manual wounds may also be unstable at low intraocular pressure, allowing bacteria into the eye from the conjunctival sac, causing endophthalmitis. If the wound has a trapezoid structure and the inner lip is narrower than the outer, it helps to keep the corneal wound tightened. With femtolaser technology, any kind of wound with any geometry can be created with the desired size, location, and number. Peripheral localization is very important to avoid SIA.

Masket et al studied femtolaser-created corneal incisions and found that femto-wounds were more stable and easier to reproduce..$^{35}$ A multiplanar geometrical wound structure could easily be achieved. ${ }^{35}$

Femtosecond laser-created corneal wounds are selfsealing; they do not need to be hydrated at the end of surgery due to the wound structure and geometry and less stress, phacoemulsification time, and CDE during surgery. Theoretically, the better wound structure and stability cause less postoperative endophthalmitis and SIA, although this needs to be proved by peer-reviewed multicenter studies in future.

Limbal relaxing incisions (LRI) are typically created using a handheld diamond knife. The real depth of incision is difficult to control manually. Surgeons may use corneal marks for better placement of incisions, so incisions might be imprecise depending on incision depth and architecture. The Alcon-LenSx femtosecond laser has an image-guided capability which is able to control the corneal thickness measurements, the shape, placement, and incision length, width, and depth (percentage of corneal thickness). The procedure is computer-controlled, predictable, and precise. Donnenfeld and Slade are still working on the best nomogram to precisely predict the refractive effects and outcome of the arcuate corneal incisions. The surgeon can immediately open the incision after femtolaser pretreatment or can wait until the next postoperative day. This way, the surgeon can take into consideration the SIA and can topographically control at what depth the corneal wound should be opened the next day at the slit lamp. Thus, the effect of femtolaser-created arcuate corneal incisions can be titrated in order to achieve the most optimal effect.

\section{Refractive outcome}

A prospective, non-randomized study was performed in order to determine the internal aberrations in eyes treated with the femtosecond laser. The results were compared to manually executed phacoemulsification eyes. Femtolaser anterior capsulotomies were performed in 48 eyes and manual CCC in 51 eyes. The results revealed that femtolaser capsulotomy induced significantly less internal aberrations as measured by the Nidek optical path difference (OPD) scan aberrometer (Nidek Inc., Fremont, CA, USA). ${ }^{5}$ The main outcome measures during the study were the postoperative visual acuity (uncorrected, best spectacle corrected), residual refraction, ocular and internal aberrations (the lower the value, the better the result), the Strehl ratio (the higher the better; to quantify the effect of wavefront aberration on image quality $=$ quality of vision), and modulation transfer function (MTF; to measure the sharpness of the image created by the IOL; the higher the better). There was no statistically difference found between the groups regarding postoperative refraction and uncorrected and best-corrected distant visual acuity. The femtosecond-treated eyes showed significantly better quality of vision postoperatively. The femtosecond-treated eyes had lower values of intraocular vertical tilt $\left(Z_{1}^{-1}\right)$ and coma aberrations $\left(Z_{3}^{-1}\right)$, higher Strehl ratios, and higher MTF values at all measured cycles per degree $(P<0.05){ }^{5}$ In summary, femtolaser-created anterior capsulotomy eyes showed better quality of vision and significantly fewer internal aberrations compared to traditional manual capsulorhexis.

In another prospective, randomized study carried out by Filkorn et a ${ }^{18}$ results showed a significantly lower mean absolute error after FLACS. This was more significant in eyes with shorter and longer AL. There was no statistically significant difference regarding the refractive outcome of FLACS and manual phacoemulsification. ${ }^{18}$ Roberts et al also found no statistically significant difference for refractive outcome ${ }^{36}$ and Palanker et al found a similar refractive outcome. ${ }^{31}$

Szigeti et al found that $5.5 \mathrm{~mm}$ central femtolaser-assisted capsulorhexis tilt and decentration was better compared to the $6.0 \mathrm{~mm}$ diameter group. ${ }^{13}$ The authors implanted a $5.0 \mathrm{~mm}$ diameter single-optic accommodating a Crystalens AT-50AO IOL (Bausch \& Lomb Incorporated). The study showed no difference between the groups in terms of uncorrected and best corrected near and far visual acuities. ${ }^{13}$

Lawless studied a cohort of 60 and 29 eyes operated on with FLACS and manual phacoemulsification, respectively. ${ }^{37}$ A diffractive multifocal IOL was implanted (Restor SN6AD1; Alcon Laboratories Inc.). The results showed no difference in the mean postoperative spherical equivalent refraction between the two patient groups. ${ }^{37,38}$

It should be emphasized that expectations are set very high with femtosecond laser technology. It is to be expected, but the method does not differ as much from traditional manual phacoemulsification as phacoemulsification differed from extracapsular cataract removal. It helps to create consistent 
and customized steps of cataract surgery, which render the method safer and more predictable than ever before. In this way, the offer of premium lenses (aspheric, toric, multifocal, multifocal-toric, accommodating, adjustable, etc) can be optimally used for the benefit of patients and ophthalmologists as well.

\section{Endothelial effects}

Following manual phacoemulsification, an average of $8.5 \%$ cell loss has been reported in the literature 12 months postoperatively. The majority of the cell loss occurs during the first 6 weeks $(\sim 7.5 \%)$, followed by $\sim 1 \%$ loss during the rest of the first postoperative follow-up year. ${ }^{8}$

Takacs et al performed a prospective randomized study with FLACS compared to manual phacoemulsification. ${ }^{8}$ The authors examined the effect of femtolaser treatment on corneal thickness, corneal volume stress index, and endothelial density, and found significantly better results in the early postoperative period in favor of the femtolaser-treated group regarding corneal thickness (580 versus $610 \mu \mathrm{m})(P \leq 0.05)$. The volume stress index was also found to be statistically significantly lower among the femtolaser-treated eyes and the same was found for endothelial density. ${ }^{8}$ The authors concluded that on the first postoperative day, femtolaser-treated eyes showed significantly lower corneal thickness compared to the manual group; this difference disappeared 1 week and 1 month following surgery. The reasoning behind this is the shorter phacoemulsification time with less CDE.

Abell et al found no difference in endothelial cell loss 3 weeks after FLACS or manual phacoemulsification. ${ }^{39}$

\section{Macular effects}

Twenty eyes of 20 patients underwent femtolaser treatment and 20 eyes of 20 other patients received traditional phacoemulsification with manual CCC in a study on macular thickness. Macular thickness and volume were assessed by OCT (Zeiss GmbH, Jena, Germany) 1 week and 1 month, postoperatively. Outcome measurements were retinal thickness assessed by the OCT in three macular areas and total macular volume at 1 week and 1 month, postoperatively. Secondary outcome measures were changes in retinal thickness at 1 week and 1 month, postoperatively, in comparison with preoperative retinal thickness values. Multivariable statistical modeling showed significantly lower macular thickness in the inner retinal ring in the femtolaser-treated group after adjusting for age and preoperative thickness across the whole time course $(P=0.002)$. In the traditional phacoemulsification group, the inner macular layer was significantly thicker at 1 week; after 1 month, this difference was no longer statistically significant. ${ }^{6,7}$ Thus, regarding safety issues, femtosecond laser-assisted cataract eyes do not differ in terms of macular thickness compared to traditional ultrasound phacoemulsification; on the other hand, results are somewhat better regarding the thickness of the inner retinal layer in the first week following surgery.

\section{Femtosecond laser cataract surgery in difficult cases}

Nagy et al have reported successful femtolaser application in cases of ocular trauma with anterior capsular rupture. ${ }^{16}$ Femtolaser capsulotomy helps to create a central and roundshaped capsulotomy without propagating the traumatic capsular rupture to the posterior capsule, thus enabling the surgeon to implant the PCL into the capsular sack. In this way, a more favorable visual outcome can be expected and patients may return to work earlier. ${ }^{16}$

Femtolaser technology was also successfully applied in eyes with angle-closure glaucoma; in the reported case, the ACD was $1.1 \mathrm{~mm}$. With the aid of the inbuilt OCT, a safe and guaranteed size of capsulotomy could be achieved with efficient fragmentation. In the literature, for non-dilating pupils, a Malyugin ring was first used during femtolaser cataract surgery by our working team, and this was published in 2013. ${ }^{17}$

Femtosecond laser capsulotomy is also possible in eyes with penetrating keratoplasty (PKP) in their anamnesis. Circular scar lines of PKP usually do not disturb femtocapsulotomy, because the scar line is around $7.0 \mathrm{~mm}$ in diameter, while the capsulorhexis is below $5.0 \mathrm{~mm}$. Also, the docking procedure is no more complicated than the primary FLACS procedures. ${ }^{15}$

Femtolaser capsulotomy is also applicable in eyes with keratoconus, even in advanced cases (data not shown).

Schultz et al reported a successful FLACS procedure in a cataractous decentered lens of a child with Marfan syndrome. The advantage of femtolaser capsulotomy in this case was clear and has life-long importance. ${ }^{40}$

Pediatric cataract may also be an important field of application for femtolasers due to the high elasticity of pediatric crystalline lenses. Well-centered and capsulorhexes of a predictable size are of high importance and posterior capsulotomy especially may render the method even more useful. Dick and Schultz ${ }^{41}$ reported successful pediatric cataract cases with four infants. The authors measured the capsulotomy diameter to be slightly larger than expected due to the higher elasticity of infant capsules. ${ }^{41}$ 


\section{What can research do to help the femtolaser cataract surgeon based on the newest developments?}

Recently an article was published by Schultz et al about the increase of prostaglandin- $\mathrm{E}_{2}\left(\mathrm{PGE}_{2}\right)$ concentration in the aqueous humor. ${ }^{42}$ The authors found an immediate rise of $\mathrm{PGE}_{2}$ in the aqueous humor using an enzyme-linked immunoassay method. This $\mathrm{PGE}_{2}$ increase may contribute to the meiosis effect that was described in one-third of cases by experienced surgeons, especially during the learning curve. Besides the mechanical pressure effect of the patient interface, bubble formation in the anterior chamber may also contribute to the mechanical effects in increasing the $\mathrm{PGE}_{2}$ level in the aqueous humor.

Prostaglandins are high potential bioregulatory substances and are synthesized in the cyclo-oxygenase pathway from arachidonic acid. Within the eye, the main sources of prostaglandins are the non-pigmented epithelial layer of ciliary bodies. Mechanical and thermal stimuli increase the level of prostaglandins in the aqueous humor according to Cole and Unger $^{43}$ and Mailhöfner et al. ${ }^{44}$

In previous studies, Gimbel found that pupillary constriction was reduced in patients receiving a preoperative NSAID regimen. ${ }^{45}$ Bucci et al also found that $\mathrm{PGE}_{2}$ levels are reduced by using NSAID drops prior to cataract surgery. ${ }^{46}$

So based on the Schultz study, it can be presumed that femtolaser pretreatment increases the levels of $\mathrm{PGE}_{2}$ in the aqueous humor, and patients should be pretreated with NSAID medication prior to surgery, and pupil dilation with combined drops should also be started earlier, compared to in normal phacoemulsification. In the case of perioperative pupillary miosis, intracameral epinephrine has been found to be useful for controlling pupil diameter.

\section{Conclusion}

The most important advantage of femtolaser cataract technology is that all the steps can be customized, delivering higher accuracy, repeatability, and consistency in results compared to in traditional phacoemulsification. Femtolaser treatment of the crystalline lens increases safety, efficacy, and predictability of the surgery. Surgical skill and wisdom are still needed to avoid possible complications that could arise during lens surgery. During well-prepared surgeries (thorough patient information and selection, proper patient interface insertion, well-designed and performed capsulotomy, lens fragmentation/liquefaction, corneal wound, and astigmatism correction), the safety of refractive cataract surgery increases and all the advantages of premium lenses can be achieved and passed on to patients. Pricing is an important factor in how quickly the procedure will spread, but with the aging population and the increasing numbers of cataract lens surgeries, wide acceptance and use is expected in the near future.

The intraoperative complication rate after the learning curve seems to be lower but at least comparable to standard manual phacoemulsification. NSAID drops are advised for use preoperatively to keep the pupil well-dilated during surgery. Increased safety, promising results with ELPo, higher predictability, and surgical consistency may render the method generally acceptable soon.

In the future, compound femtolaser equipments are to be expected, which may be applicable for corneal and lens procedures as well. Presbyopia correction is also promising and is still under investigation. At present, higher predictability and safety are the main issues of FLACS. However, we still await the results of evidence-based medicine. The European Society of Cataract and Refractive Surgeons (ESCRS) decided in 2013 to launch and to conduct a study for the complication rate of femtolaser cataract surgeries during a European-based prospective 1-year study. The ophthalmic community is looking forward to seeing the results and other peer-reviewed literature data.

\section{Disclosure}

The author reports no conflicts of interest in this work.

\section{References}

1. Koopman S [webpage on the Internet]. Cataract Surgery Devices - Global Pipeline Analysis, Competitive Landscape and Market Forecasts to 2017 [January 2012]. London, UK: GlobalData. Available at: https://www. asdreports.com/shopexd.asp?id=25116. Accessed March 18, 2014.

2. Nagy ZZ, Takacs A, Filkorn T, Sarayba M. Initial clinical evaluation of intraocular femtosecond laser in cataract surgery. J Refract Surg. 2009;25:1053-1060.

3. Nagy ZZ, Kranitz K, Takacs AI, et al. Comparison of intraocular lens decentration parameters after femtosecond and manual capsulotomies. J Refract Surg. 2011;27:564-569.

4. Kranitz K, Takacs A, Mihaltz K, Kovács I, Knorz MC, Nagy ZZ. Femtosecond laser capsulotomy and manual continuous curvilinear capsulorhexis parameters and their effects on intraocular lens centration. $J$ Refract Surg. 2011;27:558-563.

5. Mihaltz K, Knorz MC, Alio JL, et al. Internal aberration and optical quality after femtosecond laser anterior capsulotomy in cataract surgery. J Refract Surg. 2011;27:711-716.

6. Ecsedy M, Mihaltz K, Kovacs I, Takács A, Filkorn T, Nagy ZZ. Effect of femtosecond laser cataract surgery on the macula. J Refract Surg. 2011;27:717-722.

7. Nagy ZZ, Ecsedy M, Kovacs I, et al. Macular morphology assessed by optical coherence tomography image segmentation after femtosecond laser-assisted and standard cataract surgery. $J$ Cataract Refract Surg. 2012;38:941-946.

8. Takacs AI, Kovacs I, Mihaltz K, Filkorn T, Knorz MC, Nagy ZZ. Central corneal volume and endothelial cell count following femtosecond laser-assisted refractive cataract surgery compared to conventional phacoemulsification. J Refract Surg. 2012;28:387-391. 
9. Nagy ZZ, Filkorn T, Takacs AI, et al. Anterior segment OCT imaging after femtosecond laser cataract surgery. J Refract Surg. 2013; 29:110-112.

10. Nagy ZZ. Advanced technology IOL-s in cataract surgery: pearls for successful femtosecond cataract surgery. Int Ophthalmol Clin. 2012; 52:103-114.

11. Kranitz K, Mihaltz K, Sandor GL, Takacs A, Knorz MC, Nagy ZZ. Intraocular lens tilt and decentration measured by Scheimpflug camera following manual or femtosecond laser-created continuous circular capsulotomy. J Refract Surg. 2012;28:259-263.

12. Alio JL, Abdou AA, Soria F, et al. Femtosecond laser cataract incision morphology and corneal higher-order aberration analysis. J Refract Surg. 2013;29:590-595.

13. Szigeti A, Kranitz K, Takacs AI, Mihaltz K, Knorz MC, Nagy ZZ. Comparison of long-term visual outcome and IOL position with a single-optic accomodating IOL after 5.5 to $6.0 \mathrm{~mm}$ femtosecond laser capsulotomy. J Refract Surg. 2012;28:609-613.

14. Nagy ZZ, et al. Complications of femtolaser assisted cataract surgery. J Cataract Refract Surg. 2014;40:20-28.

15. Nagy ZZ, Takacs AI, Filkorn T, et al. Laser refractive cataract surgery with a femtosecond laser after penetrating keratoplasty: case report J Refract Surg. 2013;29:8.

16. Nagy ZZ, Kranitz K, Takacs A, Filkorn T, Gergely R, Knorz MC. Intraocular femtosecond laser use in traumatic cataracts following penetrating and blunt trauma. J Refract Surg. 2012;28:151-153.

17. Kranitz K, Takacs AI, Gyenes A, et al. Femtosecond laser-assisted cataract surgery in management of phacomorphic glaucoma. J Refract Surg. 2013;29:645-648.

18. Filkorn T, Kovacs I, Takacs A, Horvath E, Knorz MC, Nagy ZZ. Comparison of IOL power calculation and refractive outcome after laser refractive catarct surgery with a femtosecond laser versus conventional phacoemulsification. J Refract Surg. 2012;28:540-544.

19. Talamo JH, Gooding P, Angeley D, et al. Optical patient interface in femtosecond laser-assisted cataract surgery. Contact corneal applanation versus liquid immersion. J Cataract Refract Surg. 2013;39:501-510.

20. Kerr NM, Abell RG, Voth BJ, Toh T. Intraocular pressure during femtosecond laser pretreatment of cataract. J Cataract Refract Surg. 2013;39:339-342.

21. Roberts T, Sutton G, Lawless M, Jindal-Bali S. Capsular blockage syndrome associated with femtosecond laser-assisted cataract surgery. J Cataract Refract Surg. 2011;37:2068-2070.

22. Laksminarayanan V, Enoch JM, Raasch T, Crawford B, Nygaard RW. Refractive changes induced by intraocular lens tilt and longitudinal displacement. Arch Ophthalmol. 1986;104:90-92.

23. Erickson P. Effects of intraocular lens position errors on postoperative refractive error. J Cataract Refract Surg. 1990;16:305-311.

24. Atchinson DA. Refractive error induced by displacement of intraocular lenses within the pseudophakic eye. Optom Vis Sci. 1989; 66:146-152.

25. Kozaki J, Tanihara H, Yasuda A, Nagata M. Tilt and decentration of the implanted posterior chamber intraocular lens. J Cataract Refract Surg. 1991;17:592-595.

26. Korynta J, Bok J, Cendelin J. Change in refraction induced by change in intraocular lens position. J Refract Corneal Surg. 1994;10:556-564.

27. Ravalico G, Tognetto D, Palomba M, Busatto P, Baccara F. Capsulorhexis size and posterior capsule opacification. J Cataract Refract Surg. 1996;22:98-103.

Clinical Ophthalmology

\section{Publish your work in this journal}

Clinical Ophthalmology is an international, peer-reviewed journal covering all subspecialties within ophthalmology. Key topics include: Optometry; Visual science; Pharmacology and drug therapy in eye diseases; Basic Sciences; Primary and Secondary eye care; Patient Safety and Quality of Care Improvements. This journal is indexed on Submit your manuscript here: http://www.dovepress.com/clinical-ophthalmology-journal
28. Norrby S. Sources of error in intraocular lens power calculation. J Cataract Refract Surg. 2008;34:368-376.

29. Cekic O, Batman C. The relationship between capsulorhexis size and anterior chamber depth relation. Ophthalmic Surg Lasers. 1999; 30:185-190.

30. Friedman NJ, Palanker DV, Schuele G, et al. Femtosecond laser capsulotomy. J Cataract Refract Surg. 2011;37:1189-1198.

31. Palanker DV, Blumenkrantz MS, Andersen D, et al. Femtosecond laserassisted cataract surgery with integrated optical coherence tomography. Sci Transl Med. 2010;2:58-85.

32. Conrad-Hengerer I, Hengerer FH, Schultz T, Dick HB. Effect of femtosecond laser fragmentation on effective phacoemulsification time in cataract surgery. J Refract Surg. 2012;28:879-883.

33. Taban M, Behrens A, Newcomb RL, et al. Acute endophthalmitis following cataract surgery: a systematic review of hte literature. Arch Ophthalmol. 2005;123:613-620.

34. Ernest PH, Lavery KT, Kiessling LA. Relative strength of scleral corneal and clear corneal incisions constructed in cadaver eyes. J Cataract Refract Surg. 1994;20:626-629.

35. Masket S, Sarayba M, Ignacio T, Fram N. Femtosecond laser-assisted cataract incisions: architectural stability and reproducibility. J Cataract Refract Surg. 2010;36:1048-1049.

36. Roberts TV, Lawless M, Chan CC, et al. Femtosecond laser cataract surgery: technology and clinical practice. Clin Experiment Ophthalmol. 2013;41:180-186.

37. Lawless M, Bali SJ, Hodge C, Roberts TV, Chan C, Sutton G. Outcomes of femtosecond laser cataract surgery with a diffractive multifocal intraocular lens. J Refract Surg. 2012;28:859-864.

38. Roberts TV, Lawless M, Bali SJ, Hodge S, Sutton G. Surgical outcomes and safety of femtosecond laser cataract surgery: a prospective study of 1500 consecutive cases. Ophthalmology. 2013;120:227-233.

39. Abell RG, Kerr NM, Vote BJ. Toward zero effective phacoemulsification time using femtosecond laser pretreatment. Ophthalmology. 2013;120:942-948.

40. Schultz T, Ezeanoskie E, Dick HB. Femtosecond laser-assisted cataract surgery in pediatric Marfan syndrome. J Refract Surg. 2013;29:650-652.

41. Dick HB, Schultz T. Femtosecond laser-assisted cataract surgery in infants. J Cataract Refract Surg. 2013;39:665-668.

42. Schultz T, Joachim SC, Kuehn M, Dick BH. Changes in prostaglandin levels in patients undergoing femtosecond laser-assisted cataract surgery. J Refract Surg. 2013;29:742-747.

43. Cole DF, Unger WG. Prostaglandins as mediators for the responses of the eye to trauma. Exp Eye Res. 1973;17:357-368.

44. Mailhöfner C, Schlötzer-Schrehardt U, Gühring H, et al. Expression of cyclo-oxygenase-1 and -2 in normal and glaucomatous human eyes. Invest Ophthalmol Vis Sci. 2001;42:2616-2624.

45. Gimbel HV. The effect of treatment with topical nonsteroidal antiinflammatory drugs with and without intraoperative epinephrine on the maintenance of mydriasis during cataract surgery. Ophthalmology. 1989;96;22:585-588.

46. Bucci FA Jr, Waterbury LD. Aqueous prostaglandin E(2) of cataract patients at trough ketorolac and bromfenac levels after 2 days dosing Adv Ther. 2009;26:645-650.

PubMed Central and CAS, and is the official journal of The Society of Clinical Ophthalmology (SCO). The manuscript management system is completely online and includes a very quick and fair peer-review system, which is all easy to use. Visit http://www.dovepress.com/ testimonials.php to read real quotes from published authors. 